\title{
Modulated Nanostructure Characterization Using Aberration Corrected STEM
}

Ray Carpenter ${ }^{1}$, Ronit Sawant ${ }^{2}$ and Toshihiro Aoki ${ }^{3}$

${ }^{1}$ Arizona State University, Mesa, Arizona, United States, ${ }^{2}$ Arizona State University, Tempe, Arizona, United States, ${ }^{3}$ University of California - Irvine, Irvine, California, United States

Modulated nanostructures consist of short wavelength periodic composition fluctuations in metal, semiconductor, or ceramic alloys and they have strong effects on alloy properties. Their existence is often attributed to spinodal decomposition (1). The early stage nanostructure consists of periodic composition fluctuations whose amplitude and wavelength increase as the transformation progresses to equilibrium. Typical wavelength ranges 5 to $15 \mathrm{~nm}$ (2), but the initially small composition amplitudes must become large as the reaction progresses according to system thermodynamics. The constituent atoms are generally of different sizes so the fluctuations result in a tessellated strain field oriented in the soft directions of the host crystal lattice. Direct measurements of the composition fluctuation amplitudes and wavelengths are the best method to study the reaction kinetics. Bentley's comprehensive review (3) shows that diffraction contrast imaging/selected area diffraction produced much useful information about the periodicity and strain fields in modulated nanostructures, including defects in the fluctuation periodicity caused by more familiar lattice defects such as grain boundaries, but little direct information about the amplitude of the fluctuations, due to instrumental limitations of the time. Since then the development of field emission sources (4), aberration correctors (5), and ADF imaging for STEM microscopes should provide the necessary resolution to examine the shape of diffuse interfaces and the amplitudes of modulations directly.

We used a conventional TEM/STEM (not aberration corrected) with a Schottky FEG source to examine composition modulations in $\mathrm{Al}_{0.4} \mathrm{Ga}_{0.6} \mathrm{~N}$ layers grown by molecular beam epitaxy. The growth temperature was $750 \mathrm{C}$, high enough to insure atomic mobility to form a modulated structure (6). ADF images showed that modulated structure with an average wavelength of $5.9 \pm 1.4 \mathrm{~nm}$ existed in the layers. We could establish the $\mathrm{Ga}$ and $\mathrm{Al}$ rich regions in the modulations using nanospectroscopy, but the spatial resolution was insufficient for quantitative analysis. We have now examined these layers in an aberration corrected D-STEM microscope. Figure 1 shows a typical modulated structure image. The modulation is one-dimensional, and the modulation wave vector is nearly perpendicular to [0001]. The layer has the wurtzite structure, and the basal plane spacing is modulated by the composition variations. The modulations are not as regular as one would expect from theory. The places where dark regions (Al-rich) transform to light regions (Ga-rich) probably occur because the wavelength was growing as the layer formed during synthesis at elevated temperature. This aspect of the nanostructure is in common with those of many metal alloy modulated structures reviewed by Bentley. Whether these composition modulations are one, two, or three dimensional has been a topic of discussion. Many early investigations used satellites in X-ray diffraction patterns or images from thick specimens to conclude that they were multidimensional. We have so far only observed one dimensional composition modulations. Figure 2 shows the $\mathrm{Al}$ and Ga composition variations along [0001] calculated from an EELS line scan of one region of this specimen. A similar plot for $\mathrm{N}$ showed little variation with distance, indicating small thickness variation. The left side of the plot shows fairly well behaved composition fluctuations with a wavelength of about $5 \mathrm{~nm}$, but these become increasingly irregular approaching the right side. The irregularity is caused by the presence of a grain boundary and some nearby lattice defects. The presence of defects disrupts the periodicity of the composition modulations. Note that the composition of the regular Alrich modulations on the left is nearly AlN, containing perhaps $5 \% \mathrm{Ga}$, while the Ga-rich part of the modulations contain 20 to $35 \% \mathrm{Al}$. This is an unexpected result. If the composition modulations result from a miscibility gap in the Al-Ga-N diagram one would expect compositions of the modulations to lie on a tie line connecting AlN and GaN, because no ternary compounds have been reported for the ternary system. The presence of large 
amounts of $\mathrm{Al}$ in the Ga-rich part of the modulations indicates that the end of the tie line containing Ga ends near the middle of the ternary diagram and not at GaN. Further, note that the subpeaks of the composition plots in the Ga-rich part of the composition modulations are very sharp and approximate the basal plane spacing of the specimen. This indicates that these regions may be chemically ordered. The maximum rate of concentration change at the diffuse interfaces is about $17 \mathrm{At} . \% / \mathrm{nm}$, which is much slower than the rate of change at "chemically sharp" interfaces. These results showed that our current aberration corrected monochromated STEM instruments are quite capable for characterizing modulated nanostructures down to atomic level. We are also examining modulated nanostructures in the $\mathrm{Cu}$-Ti system that contains an asymmetric miscibility gap and in the Au-Pt system that contains a nearly symmetric miscibility gap. An important objective for these systems is determining the rate of composition amplitude change with isothermal aging time, which will allow direct comparison of experimental aging kinetics with spinodal decomposition rate theory (1). This can be done using EELS, but it would simpler to use HAADF $\mathrm{Z}$ contrast images if an accurate relationship can be established between image contrast and composition for the same area determined by EELS. HAADF image contrast depends on $\mathrm{Z}^{\mathrm{n}}$, where $\mathrm{Z}$ is the atomic number of the specimen and thus directly related to composition, and $\mathrm{n}$ also depends on $\mathrm{Z}$. Values for $\mathrm{n}$ for single atom HAADF images of elements of interest here range from about 1.6 to 1.9 at 100 to $200 \mathrm{kV}(7,8)$. We are comparing our EELS results to composition predictions from HAADF image intensities using these theoretical values for $n$.

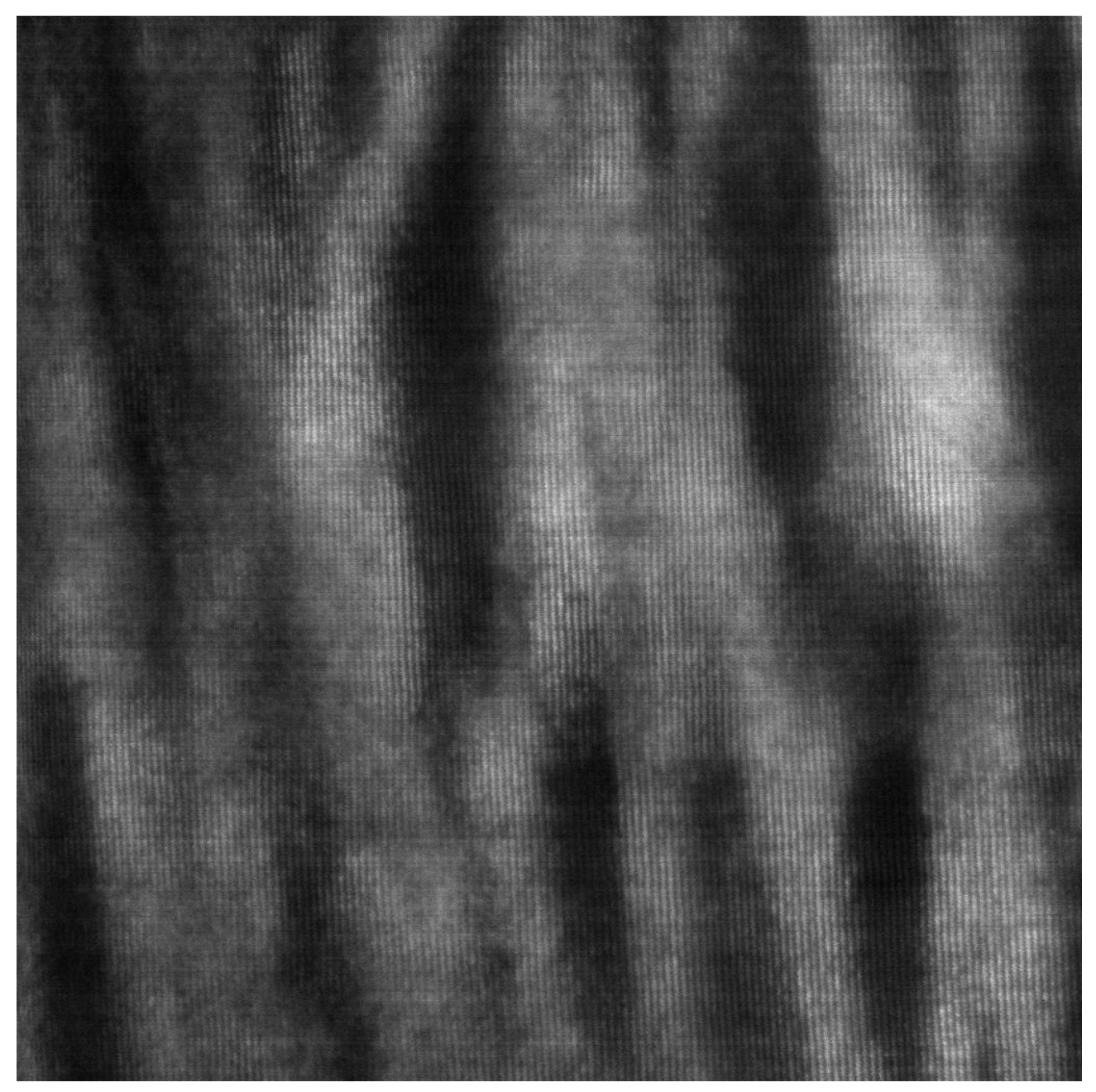


Figure 1. HAADF STEM image of modulated nanostructure in AlGaN layer grown by molecular beam epitaxy at 750C. The average wavelength is $5.9 \mathrm{~nm}$. The layer is wurtzite structure. Basal planes are in contrast. Beam direction near $<10-10\rangle$ slightly rotated around $<0002>$. $100 \mathrm{kV}$.

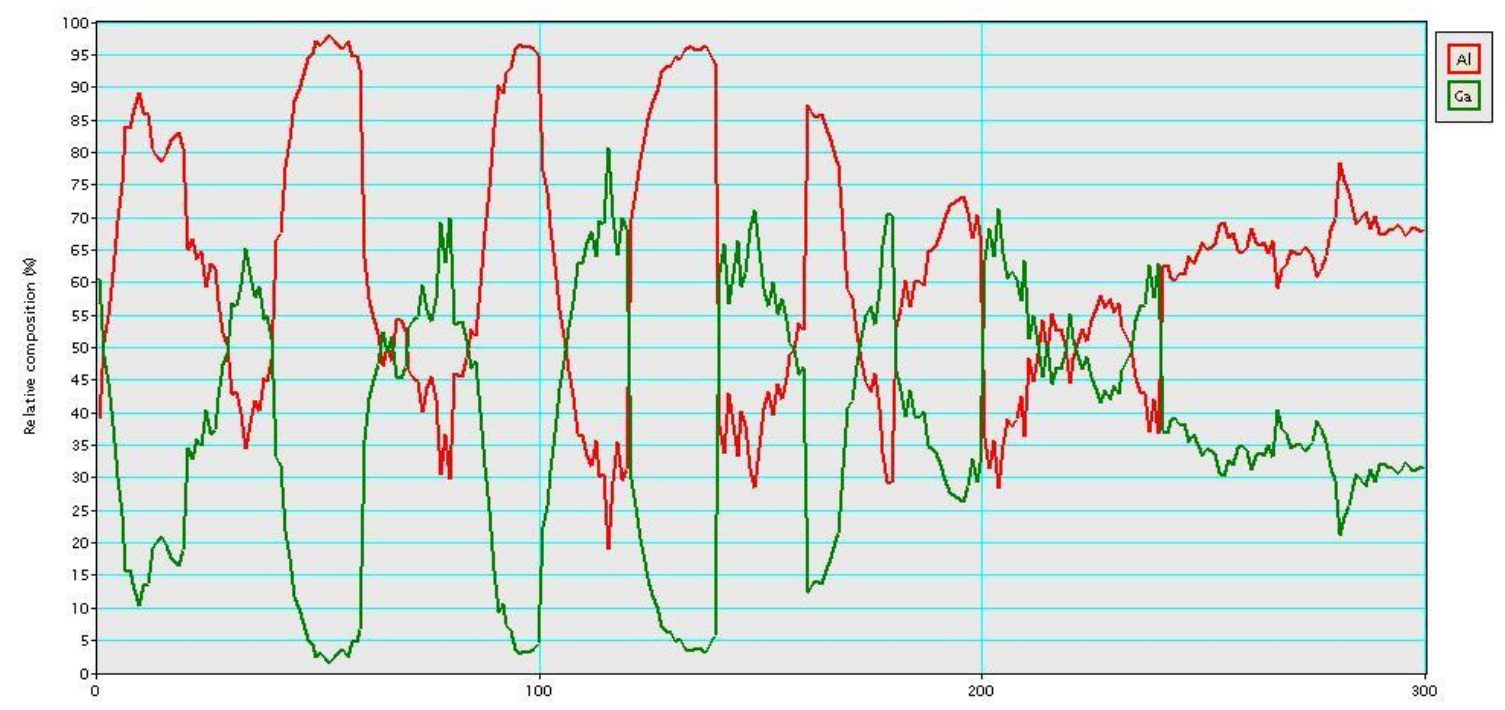

Figure 2. EELS line scan across modulated structure in AlGaN layer grown by molecular beam epitaxy at 750 C. Scan direction is $\langle 0002\rangle$. The irregularities in the composition modulations on the right are caused by lattice defects including a grain boundary in the layer at that location. Horizontal distance scale in Angstroms. Vertical scale in At. \%. Red=Al. Green=Ga.

\section{References}

1. J. W. Cahn (1962), Acta Met.10, 179-183.

2. R. W. Carpenter (1967), Acta Met.15, 1567-1572.

3. J. Bentley (1989), Ultramicros. $\underline{30}, 157-171$.

4. A. V. Crewe et al (1975), p. 47 in Physical Aspects of Electron Microscopy and Microbeam Analysis, ed. by Siegel and Beaman, Wiley, New York.

5. O. L. Krivanek et al (2008), Ultramicros.108, 179-195.

6. A. Wise et al (2008), App. Phy. Lett. 92, 261914.

7. M. M. J. Treacy (2011), Micros. Microanal.17, 847-858.

8. P. Hartel et al (1996), Ultramicros. 63, 93-114. 